\title{
Incidencia y factores de riesgo de estenosis de la anastomosis vesicouretral en pacientes operados de prostatectomía radical extraperitoneal videoendoscópica
}

\author{
Incidence of and risk factors for vesicourethral anastomotic \\ stricture in patients that underwent video-endoscopic \\ extraperitoneal radical prostatectomy
}

José David Cabrera-Ledesma, Fátima Sandoval, Jesús Torres-Aguilar

\begin{abstract}
Resumen
ANTECEDENTES: La estenosis de la anastomosis vesicouretral es una complicación importante de la prostatectomía radical, a mediano y largo plazos, con incidencia y causalidad heterogénea.

OBJETIVO: Analizar y determinar las causas relacionadas con la estenosis de la anastomosis vesicouretral, incluidas las características propias del paciente y los factores clínicos pre, trans y posoperatorios.

MATERIALES Y MÉTODOS: Estudio de casos y controles, retrospectivo efectuado con base en la información de los expedientes de pacientes admitidos en el servicio de Urología del Hospital Juárez de México, entre los años 2010 a 2016, con diagnóstico de cáncer de próstata y prostatectomía radical extraperitoneal videoendoscópica. Para el análisis estadístico se calculó el riesgo relativo y se utilizaron las pruebas de $\chi^{2}$ y t de Student. RESULTADOS: Se registraron 74 pacientes. En 31/74 se estableció el diagnóstico de estenosis durante el seguimiento mínimo de 3 años, quienes requirieron un nuevo tratamiento endoscópico o dilataciones. Factores de riesgo: diabetes mellitus ( $p=$ $0.003)$, técnica de sutura interrumpida de anastomosis $(p=0.03)$, volumen prostático menor (media de $53 \mathrm{~g} ; \mathrm{p}=0.02)$ y radioterapia posoperatoria $(p=0.001)$.

CONCLUSIONES: La incidencia de estenosis de la anastomosis vesicouretral es elevada. Los factores de riesgo asociados incluyen: diabetes mellitus, técnica de sutura interrumpida, radioterapia coadyuvante y volumen prostático menor (53 g).

PALABRAS CLAVE: Estenosis de la anastomosis vesicouretral; prostatectomía radical; cáncer de próstata.

Abstract

BACKGROUND: Vesicourethral anastomotic stricture is an important medium-term and long-term complication of radical prostatectomy and its incidence and causality are heterogeneous.

OBJECTIVES: To analyze and determine the causes related to vesicourethral anastomotic stricture, including patient characteristics and preoperative, intraoperative, and postoperative factors.

MATERIALS AND METHODS: A retrospective case-control study was conducted that reviewed the case records of patients admitted to the Urology Service of the Hospital Juárez de México within the time frame of 2010 to 2016 that were diagnosed with prostate cancer and underwent video-endoscopic extraperitoneal radical prostatectomy. For the statistical analysis, the relative risk was calculated, and the chi-square test and Student's t test were performed.
\end{abstract}

Servicio de Urología, Hospital Juárez de México, Ciudad de México.

Recibido: febrero 2018

Aceptado: julio 2018

Correspondencia José David Cabrera Ledesma jdavidcabrera88@gmail.com

Este artículo debe citarse como Cabrera-Ledesma JD, Sandoval F, Torres-Aguilar J. Incidencia y factores de riesgo de estenosis de la anastomosis vesicouretral en pacientes operados de prostatectomía radical extraperitoneal videoendoscópica. Rev Mex Urol. 2018 julio-agosto;78(4):283-289.

DOI: https://doi.org/10.24245/revmexurol.v78i4.2023 
RESULTS: Seventy-four patients were registered. Thirty-one of them were diagnosed with vesicourethral anastomotic stricture during their minimum 3-year follow-up period. Risk factors were diabetes mellitus $(p=0.003)$, anastomotic interrupted suture technique $(p=0.03)$, lower prostate volume (53 g) $(p=0.02)$, and postoperative radiotherapy $(\mathrm{p}=0.001)$.

CONCLUSIONS: The incidence of vesicourethral anastomotic stricture was high. Diabetes mellitus, the use of interrupted sutures in the anastomosis, adjuvant radiotherapy, and lower prostate volume $(53 \mathrm{~g})$ had a statistically significant relation to the presence of stricture.

KEYWORDS: Vesicourethral junction stricture; Radical prostatectomy; Prostate cancer.

\section{ANTECEDENTES}

La estenosis de la anastomosis vesicouretral es una de las complicaciones a mediano y largo plazo de la prostatectomía radical. Después de la resección de la próstata se reconstruye el cuello vesical y se crea una anastomosis vesicouretral. La experiencia quirúrgica sugiere que se trata de un procedimiento seguro; sin embargo, la estenosis de la anastomosis vesicouretral es una complicación significativa. El padecimiento puede originar síntomas urinarios obstructivos como: disminución del calibre y fuerza del chorro urinario, urgencia, vaciamiento incompleto, incluso retención aguda de orina en algunos pacientes. La incidencia varía según la población de estudio, el cirujano y las técnicas quirúrgicas, entre otros factores; por tanto, efectuar este tipo de estudios resulta importante para valorar la evolución de los pacientes. ${ }^{1}$

\section{Prostatectomía radical}

La prostatectomía radical sigue siendo el tratamiento de elección en pacientes con cáncer de próstata localizado. La experiencia y capacidad del cirujano se relacionan con adecuada curación y mínimo daño colateral de los tejidos circundantes, permite la estadificación precisa del tumor mediante el examen anatomopatológico de la pieza quirúrgica y un excelente control oncológico, con una tasa aceptable de complicaciones en pacientes seleccionados de forma apropiada. Entre las complicaciones tempranas se encuentran: hemorragia, lesión rectal, vascular, ureteral y nerviosa; filtración o fístula urinaria, linfocele y problemas con la herida quirúrgica. Las complicaciones tardías más frecuentes incluyen: disfunción eréctil, incontinencia urinaria, hernia inguinal y estenosis de la anastomosis. ${ }^{1}$

\section{Estenosis de la anastomosis vesicouretral}

La incidencia de estenosis de la anastomosis vesicouretral varía en relación con diferentes factores. ${ }^{1}$ Popken y su grupo reportan una incidencia de 0.48 a $32 \% .{ }^{2}$ Un estudio efectuado en pacientes con prostatectomía radical retropúbica refiere $7.5 \%$ y en quienes se llevó a cabo prostatectomía radical asistida por robot $2.5 \%$; de éstos, $45 \%$ ameritó tratamiento con dilataciones, $10.7 \%$ uretrotomía interna y $40 \%$ múltiples procedimientos. ${ }^{3}$ El ensayo de Kostakopoulos y sus colaboradores informó una incidencia de $6 \%$ en pacientes operados de prostatectomía radical retropúbica. ${ }^{4}$

Entre los factores de riesgo de estenosis de la anastomosis vesicouretral se encuentran: extravasación de orina, sangrado transoperatorio (mayor de $1000 \mathrm{~mL}$ ) y antecedente de procedimiento transuretral, incluso se han descrito técnicas quirúrgicas, radioterapia previa, 
enfermedades microvasculares, tabaquismo, enfermedad arterial coronaria, diabetes mellitus e hipertensión arterial. ${ }^{5}$ Los principales factores predictivos independientes de estenosis incluyen: cirugía abierta, hematuria postoperatoria, retención urinaria y recurrencia del antígeno prostático; ;,5,6 incluso algunos estudios refieren la radioterapia posoperatoria como factor predictivo significativo. ${ }^{7}$

\section{Técnicas de sutura de la anastomosis} vesicouretral

La sutura interrumpida es la técnica convencional para la anastomosis vesicouretral en pacientes intervenidos de prostatectomía radical retropúbica, mientras que la sutura continua es el patrón de referencia en quienes se practica prostatectomía laparoscópica y asistida por robot. ${ }^{8}$ Estudios recientes señalan que la extravasación desde el sitio anastomótico y la de estenosis de la anastomosis vesicouretral es considerablemente mayor en pacientes a quienes se practica prostatectomía radical retropúbica versus prostatectomía radical asistida por robot. ${ }^{3,9}$ Otra investigación que comparó ambos procedimientos quirúrgicos reportó que la fuga de orina es un factor de riesgo para padecer estenosis de la anastomosis vesicouretral. ${ }^{3}$ El tiempo de retiro del catéter transuretral y la fuga de orina fueron significativamente menores en pacientes a quienes se aplicó la sutura continua en la anastomosis vesicouretral. ${ }^{8}$ Un estudio realizado en China reportó una incidencia de $1.3 \%$ de estenosis del cuello vesical con la técnica de sutura continua para anastomosis vesicouretral. ${ }^{7}$ Otros estudios que comparan las diferentes técnicas de anastomosis vesicouretral en la prostatectomía radical laparoscópica no reportan diferencias significativas para la formación de estenosis. ${ }^{10}$

El objetivo de este estudio fue: analizar y determinar las causas relacionadas con la estenosis de la anastomosis vesicouretral, incluidas las características propias del paciente y los factores clínicos pre, trans y posoperatorios.

\section{MATERIALES Y MÉTODOS}

Estudio de casos y controles, retrospectivo, llevado a cabo en el servicio de Urología del Hospital Juárez de México, entre los años 2010 a 2016. Se seleccionaron los expedientes de pacientes con diagnóstico de cáncer de próstata, intervenidos para prostatectomía radical extraperitoneal videoendoscópica, con reporte histopatológico de la pieza quirúrgica y seguimiento en consulta externa. Se identificaron los pacientes que durante su evolución posoperatoria manifestaron síntomas urinarios de almacenamiento y vaciamiento (IPSS severo), a quienes se realizó uretrocistograma o uretrocistoscopia diagnóstica para detectar algún grado de estenosis vesicouretral y recibieron tratamiento con dilataciones o endoscopia. Las variables se analizaron mediante la revisión de las notas de ingreso, procedimiento quirúrgico original, evolución intrahospitalaria y seguimiento por la consulta externa.

\section{Análisis estadístico}

Se analizaron variables independientes como: edad, tabaquismo, enfermedad arterial coronaria, diabetes mellitus, hipertensión arterial, antecedente de cirugía transuretral, concentración de antígeno prostático específico (APE) preoperatorio, riesgo ( $\mathrm{D}^{\prime} \mathrm{Amico}$ ), técnica utilizada para la anastomosis vesicouretral (continua vs interrumpida), sangrado transoperatorio $(\mathrm{mL})$ obtenido con el sistema de aspiración de material textil, tiempo transoperatorio, fuga de orina, tiempo de permanencia de la sonda, volumen prostático (pieza), estadio patológico, puntuación de Gleason, recurrencia del APE y radioterapia posoperatoria. Las variables se tabularon y analizaron con el programa Microsoft Excel ${ }^{\circledR}$ y SPSS ${ }^{\circledR}$, con pruebas de dependencia como: $\chi^{2}, \mathrm{t}$ de Student y riesgo relativo, para 
buscar la relación con la variable dependiente de estenosis de la unión vesicouretral.

\section{RESULTADOS}

Se registraron 74 pacientes intervenidos para prostatectomía radical extraperitoneal videoendoscópica. En 31/74 se estableció el diagnóstico de estenosis de la anastomosis vesicouretral durante el seguimiento mínimo de 3 años, quienes requirieron un nuevo tratamiento endoscópico o dilataciones (Cuadro 1).

La media de edad general fue de 65.2 años. En los pacientes sin y con estenosis de la unión vesicouretral se registró un promedio de edad de 66 vs 65.9 años, respectivamente (IC95\%, $\mathrm{p}=0.91$ ), sin diferencia estadísticamente significativa.

En relación con el tabaquismo, independientemente de su intensidad, se registraron 36/74 pacientes con este hábito y de éstos 17/36 tuvieron diagnóstico de estenosis de la anastomosis vesicouretral (RR: 1.28); sin embargo, no se encontró relación estadísticamente significativa entre ambas variables (IC95\%, $p=0.36$ ), al igual que con las variables de antecedente de enfermedad arterial coronaria, hipertensión arterial y cirugía transuretral endoscópica (RR: 1.2, RR: 1.2 y RR: 1.5 , con IC95\%, p = 0.81, p $=0.52$ y $\mathrm{p}=0.21$, respectivamente). La relación entre diabetes tipo 2 y estenosis mostró una dependencia significativa (IC95\%, p = 0.003), con riesgo relativo de 2.34 .

De 31 pacientes con estenosis de la unión vesicouretral, se reportaron 17 con riesgo D'Amico intermedio; sin embargo, no se encontró relación estadísticamente significativa entre el grupo de riesgo preoperatorio y el diagnóstico de estenosis de la anastomosis vesicouretral (IC95\%, $\mathrm{p}=0.40$ ).

En cuanto a la técnica de anastomosis, se practicó sutura continua en 44 pacientes e interrumpida en 30. Estos datos mostraron que la sutura continua deriva en menos posibilidad de sufrir estenosis de la anastomosis vesicouretral durante su evolución, comparada con la sutura interrumpida (RR: 0.56), con una relación estadísticamente significativa (IC95\%, p = 0.03).

De los 31/74 pacientes con estenosis de la anastomosis vesicouretral, la mayoría tuvo sangrado transoperatorio mayor de $500 \mathrm{~mL}$ (RR: 0.78), sin encontrar diferencias significativas entre ambos grupos (IC95\%, p = 0.35), al igual que con la variable de tiempo quirúrgico, 257 vs 239 minutos en sujetos sin y con estenosis, respectivamente $($ IC95\%, $\mathrm{p}=0.31)$.

A todos los pacientes se les colocó un drenaje urinario que se midió a las 24 horas del posoperatorio, observando una media en el grupo sin y con estenosis de 222 vs $315 \mathrm{~mL}$, respectivamente, sin reportar diferencia estadísticamente significativa (IC95\%, p = 0.08). En cuanto al tiempo de permanencia de la sonda (tres semanas), tampoco se observó diferencia significativa en ambos grupos (IC95\%, $\mathrm{p}=0.38$ ).

Respecto del volumen prostático en la pieza de patología, el grupo de pacientes sin estenosis registró 64 g y con estenosis 53 g, con una diferencia significativa (IC95\%, $\mathrm{p}=0.02)$.

En relación con el estadio clínico, la mayoría de los pacientes con estenosis de la anastomosis vesicouretral se encontró en estadio II posterior al tratamiento quirúrgico; sin embargo, no reportó diferencia significativa en esta variable (IC95\%, $\mathrm{p}=0.64)$ al igual que en el puntaje de Gleason definitivo (IC95\%, p = 0.52) y la recidiva bioquímica posterior al procedimiento quirúrgico $($ IC95\%, p = 0.94).

La radioterapia coadyuvante en $7 / 74$ pacientes, todos con estenosis vesicouretral, mostró dependencia o significación estadística entre las variables (IC95\%, p = 0.001), con un RR: 2.79. 
Cabrera-Ledesma JD y col. Estenosis de la anastomosis vesicouretral

Cuadro 1. Características demográficas, clínicas y resultados

\begin{tabular}{|c|c|c|c|c|c|c|}
\hline Variable & Media & $\begin{array}{c}\text { Pacientes } \\
\text { (n) }\end{array}$ & Con estenosis & Sin estenosis & $\mathbf{R R}$ & $\mathbf{p}$ \\
\hline Pacientes (n) & & 74 & 31 & 43 & & \\
\hline Edad promedio (años) & 66 & & 65.9 (años) & 66 (años) & & 0.91 \\
\hline Tabaquismo & & 36 & 17 & 19 & 1.28 & 0.36 \\
\hline Enfermedad arterial coronaria & & 2 & 1 & 1 & 1.2 & 0.81 \\
\hline Diabetes mellitus & & 11 & 9 & 2 & 2.34 & 0.003 \\
\hline Hipertensión arterial sistémica & & 21 & 10 & 11 & 1.2 & 0.52 \\
\hline Cirugía transuretral & & 8 & 5 & 3 & 1.5 & 0.21 \\
\hline Radioterapia previa & & 2 & 2 & - & 2.48 & 0.09 \\
\hline APE preoperatorio $(\mathrm{ng} / \mathrm{mL})$ & 8.85 & & $10.5(\mathrm{ng} / \mathrm{mL})$ & $15(\mathrm{ng} / \mathrm{mL})$ & & 0.02 \\
\hline $\begin{array}{l}\text { Riesgo D’Amico } \\
\text { Bajo } \\
\text { Intermedio } \\
\text { Alto }\end{array}$ & & $\begin{array}{l}22 \\
37 \\
15\end{array}$ & $\begin{array}{c}10 \\
17 \\
4\end{array}$ & $\begin{array}{l}12 \\
20 \\
11\end{array}$ & & 0.40 \\
\hline $\begin{array}{l}\text { Técnica } \\
\text { Continua } \\
\text { Interrumpida }\end{array}$ & & $\begin{array}{l}44 \\
30\end{array}$ & $\begin{array}{l}10 \\
17\end{array}$ & $\begin{array}{l}30 \\
13\end{array}$ & 0.56 & 0.03 \\
\hline Sangrado transoperatorio $(\mathrm{mL})$ & 764 & & $854(\mathrm{~mL})$ & $698(\mathrm{~mL})$ & 0.78 & 0.35 \\
\hline Tiempo transoperatorio (min) & 249 & & 239 (min) & 257 (min) & & 0.31 \\
\hline Fuga de orina $(\mathrm{mL})$ & 261 & & $315 \mathrm{~mL}$ & $222 \mathrm{~mL}$ & & 0.08 \\
\hline Tiempo con sonda (semanas) & 3.08 & & 3 (semanas) & 3.1 (semanas) & & 0.38 \\
\hline Volumen de la pieza (g) & 59 & & $53.3(\mathrm{~g})$ & $64.4(\mathrm{~g})$ & & 0.02 \\
\hline $\begin{array}{l}\text { Estadio patológico } \\
\text { I } \\
\text { II } \\
\text { III }\end{array}$ & & $\begin{array}{c}5 \\
50 \\
19\end{array}$ & $\begin{array}{c}3 \\
21 \\
7\end{array}$ & $\begin{array}{c}2 \\
20 \\
12\end{array}$ & & 0.64 \\
\hline $\begin{array}{l}\text { Puntuación de Gleason } \\
3+3 \\
3+4 \\
4+3 \\
\text { Mayor }\end{array}$ & & $\begin{array}{l}17 \\
30 \\
13 \\
14\end{array}$ & $\begin{array}{c}9 \\
11 \\
4 \\
7\end{array}$ & $\begin{array}{c}8 \\
19 \\
9 \\
7\end{array}$ & & 0.52 \\
\hline Recidiva bioquímica & & 17 & 7 & 10 & 0.98 & 0.94 \\
\hline Radioterapia posquirúrgica & & 7 & 7 & - & 2.79 & 0.001 \\
\hline
\end{tabular}

\section{DISCUSIÓN}

La incidencia de estenosis de la anastomosis vesicouretral estimada en este estudio fue mayor que en otras series; por ejemplo, un ensayo japonés reportó una incidencia de $3.3 \%$ a un año de seguimiento, ${ }^{8}$ mientras que otra serie con mayor número de pacientes, que comparó la incidencia de estenosis de la unión vesicouretral entre sujetos operados para prostatectomía radical asistida por robot versus prostatectomía radical abierta retropúbica evidenció 7.5 vs $2.1 \%$, respectivamente. ${ }^{3}$

Nuestro estudio no mostró diferencias significativas entre las medias de edad de ambos grupos 
(IC95\%, p = 0.91), similar a lo reportado por Wang y su grupo de trabajo. ${ }^{3}$

De acuerdo con nuestros resultados, 36/74 pacientes tenían antecedente de tabaquismo (RR: 1.28) y la prueba de independencia reportó una $p=0.365$; por tanto, aunque el riesgo relativo indica un aumento mínimo de padecer estenosis vesicouretral en pacientes con hábito tabáquico, no existe relación estadísticamente significativa entre ambas variables; sin embargo, otras series indican lo contrario. ${ }^{6}$

Nuestro estudio reportó 9/31 pacientes con estenosis y antecedente de diabetes tipo 2 versus 2/43 sin estenosis. Se obtuvo un RR: 2.34 y $p=$ 0.0036 con la $\chi^{2}$, lo que indica que la diabetes constituye un factor importante de estenosis, propio del paciente, que debe considerarse en el preoperatorio, con significación estadística. Sin embargo, la hipertensión arterial sistémica y la enfermedad arterial coronaria demuestran lo contario. Diversos estudios señalan resultados heterogéneos; por ejemplo, Wang no encontró relación significativa entre estenosis vesicouretral y diabetes, pero sí con hipertensión arterial sistémica, ${ }^{3}$ comparada con el estudio de Prodromos, quien reportó una relación directa entre ambos factores de riesgo con estenosis de la anastomosis vesicouretral. ${ }^{6}$

Antes de efectuar la cirugía radical se registraron 8 pacientes con antecedente de algún procedimiento endoscópico transuretral, específicamente resección transuretral de próstata; de éstos, 5/31 versus 3/43 con y sin estenosis, respectivamente, reportaron una RR: 1.5 y $\mathrm{p}=$ 0.210 , evidenciando que este factor aumenta la posibilidad de sufrir estenosis; sin embargo, no existe relación estadísticamente significativa, similar a lo reportado en la bibliografía.

Entre los factores transoperatorios, como el sangrado estimado y el tiempo quirúrgico $(p=$
0.35 y 0.31 , respectivamente) puede evidenciarse que se trata de variables independientes a la estenosis vesicouretral; no obstante, se ha reportado relación estadística importante en series más grandes. ${ }^{3,6}$

Respecto de la técnica para efectuar la anastomosis (continua versus interrumpida), se observó que la sutura continua representa un factor protector de estenosis (RR: 0.56, p = 0.03), es decir, existe una relación dependiente importante entre ambas variables. El estudio japonés que comparó la anastomosis con técnica interrumpida versus continua en pacientes con prostatectomía radical retropúbica mostró la ventaja de la sutura continua, con menor cantidad de extravasación de orina y, por ende, retiro más temprano de la sonda transuretral. Una serie que comparó la técnica abierta con sutura interrumpida versus asistida con robot y sutura continua reportó mayor incidencia de estenosis con la primera técnica, con significación estadística importante; ${ }^{8}$ sin embargo, existen investigaciones con mayor cantidad de pacientes que no señalan diferencias significativas en cuanto a la elección de la técnica, desde el punto de vista de la tasa de extravasación, tiempo de cateterización e incidencia de estenosis de la anastomosis en pacientes a quienes se les realizó prostatectomía endoscópica extraperitoneal. ${ }^{11-13}$

La fuga de orina (drenaje en las primeras 24 horas) y el tiempo de permanencia del catéter transuretral son factores posoperatorios sin relación estadísticamente significativa con la de estenosis de la unión uretrovesical (IC95\%, $p=0.08$ ), lo que coincide con otros estudios. . $^{3,14,15}$

El volumen prostático posoperatorio fue menor en pacientes con estenosis; sin embargo, otras investigaciones no reportan relación alguna, al igual que el estadio patológico definitivo.,16 
Cabrera-Ledesma JD y col. Estenosis de la anastomosis vesicouretral

\section{CONCLUSIÓN}

Este estudio demuestra que el factor preoperatorio con mayor relación con la estenosis de la unión vesicouretral es el antecedente de diabetes mellitus tipo 2 y la técnica de sutura continua representa un factor protector significativamente relacionado con la coexistencia o no de la estenosis de la unión vesicouretral. Incluso los factores posoperatorios de menor volumen prostático y radioterapia coadyuvante tienen signifcanción estadística con la estenosis de la unión vesicouretral.

\section{REFERENCIAS}

1. Su LM, Smith JA.Prostatectomía radical por vía laparoscópica y con asistencia robótica y vaciamiento pelviano. En: Urology. 10a ed. Wein A, Kavoussi L, Novick A, Martin A, Peters $\mathrm{G}$, editors. México: Editorial Médica Panamericana, 2015; (cap 103):2852-2871.

2. Popken G, Sommerkamp H, Schulte-Seemann W, Watteraurer $U$, Katzenwadel A. Anastomotic stricture after radical prostatectomy. Incidence, findings and treatment. Eur Urol 1998;33:382-6.

3. Wang $R$, et al. Risk factors and quality of life for post prostatectomy vesicourethral anastomotic stenosis. DOI: https:// doi.org/10.1016/j.urology.2011.07.1383

4. Kostakopoulos A, et al. Vesicourethral anastomotic strictures after radical retropubic prostatectomy: The experience of a single institution. DOI: https://doi. org/10.1159/000075267

5. Zhang $\mathrm{CY}$, et al. Outcome of nephrostomy balloon dilation for vesicourethral anastomotic strictures following radical prostatectomy: A retrospective study. DOI: 10.4103/1008$682 X .122348$
6. Borboroglu PG, et al. Risk factors for vesicourethral anastomotic stricture after radical prostatectomy. Urology 2000;56:96-100.

7. Ouzaid I, et al. Anastomotic stricture after mini-invasive radical prostatectomy with Van Velthoven running suture: Incidence, predictive factors and treatment. DOI: https:// doi.org/10.1016/j.juro.2011.02.2622

8. Matsuyama I, et al. Running suture versus interrupted suture for vesicourethral anastomosis en retropubic radical prostatectomy. A randomized study. DOI: https://doi. org/10.1111/iju.12667

9. Berlin JW, et al. Voiding cystourethrography after radical prostatectomy: Normal findings and correlation between contrast extravasation and anastomotic strictures. DOI: 10.2214/ajr.162.1.8273697

10. Teber $D$, et al. Analyses of three different vesicourethral anastomotic techniques in laparoscopic radical prostatectomy. DOI: https://doi.org/10.1007/s00345-008-0281-0

11. Poulakis $\mathrm{V}$, et al. Vesicourethral anastomosis during endoscopic extraperitoneal radical prostatectomy: a prospective comparison between the single-knot running and interrupted technique. DOI: https://doi.org/10.1016/j. urology.2006.08.1063

12. Yang J, et al. Continuous suture of a single absorbable suture: a new simplified vesicourethral anastomosis technique in laparoscopic radical prostatectomy. DOI: 10.9738/ INTSURG-D-13-00124.1

13. Besarani $D$, et al. Bladder neck contracture after radical retropubic prostatectomy. DOI: https://doi.org/10.1111/ j.1464-410X.2004.05151.x

14. Sano T, et al. Relationship between type of suture and anastomotic stricture after radical prostatectomy. Hinyokika Kiyo 2010;56:95-98.

15. Ozu C, et al. Radical retropubic prostatectomy with running vesicourethral anastomosis and early catheter remove. Our experience. DOI: https://doi.org/10.1111/j.14422042.2009.02281.x

16. Sandhu JS, et al. Age, obesity, medical comorbidities and surgical technique are predictive of symptomatic anastomotic stricture after contemporary radical prostatectomy. DOI: https://doi.org/10.1016/j.juro.2011.02.003 